\section{Full cystic remnant ductoscopy: a novel criterion for defining completeness in direct cholangioscopy?}

\section{To the Editor,}

An 81-year-old female patient with a remote history of cholecystectomy was referred for endoscopic retrograde cholangiopancreatography (ERCP) due to signs of cholangitis (bilirubin $1.8 \mathrm{mg} / \mathrm{dL}$, gamma-glutamyltranspeptidase 327 $\mathrm{U} / \mathrm{L}, \mathrm{C}$-reactive protein $9.2 \mathrm{mg} / \mathrm{dL}$ ) and marked intra- and extrahepatic cholestasis on abdominal ultrasound. After an uncomplicated wire-guided biliary access, the common bile duct (CBD) proved dilated up to $25 \mathrm{~mm}$ with a large stone in its middle portion up to $20 \mathrm{~mm}$. (Fig. 1A - note limited cholangiogram due to acute cholangitis with putrid discharge). Biliary drainage using a 10-Fr biliary plastic stent was established after medium-size papillotomy, and cholangitis subsided in a timely fashion. Repeat ERCP with scheduled direct cholangioscopy (DC)-guided electrohydraulic lithotripsy was performed 6 weeks later. Of note, the large bile duct stone had apparently disintegrated under stent treatment, which is occasionally observed in clinical practice [1,2]. Given an a priori high probability for fluoroscopy-negative residual stone disease, the patient nonetheless underwent endoscopic papillary large-balloon dilation up to $15 \mathrm{~mm}$ followed by an uncomplicated freehand intubation using an ultra-slim upper endoscope (outer diameter $5.9 \mathrm{~mm}$ ). The cystic duct (CD) remnant proved low-inserting (Fig. 1B - lower left) and was first intubated up to its blind end (Fig. 1C). In addition, ancillary injection of contrast media through the endoscope's working channel firmly excluded a chronic cystic duct leakage (Fig. 1D). Next, the CBD, or more precisely, common hepatic duct was intubated up to a full endoscopic visualization of the liver hilum without evidence for residual stone material (Fig. 1E) Furthermore, limited intrahepatic cholangioscopy was accomplished up to secondary-order ducts, likewise excluding remnant intrahepatic stones. (Fig. 1F, 1G).

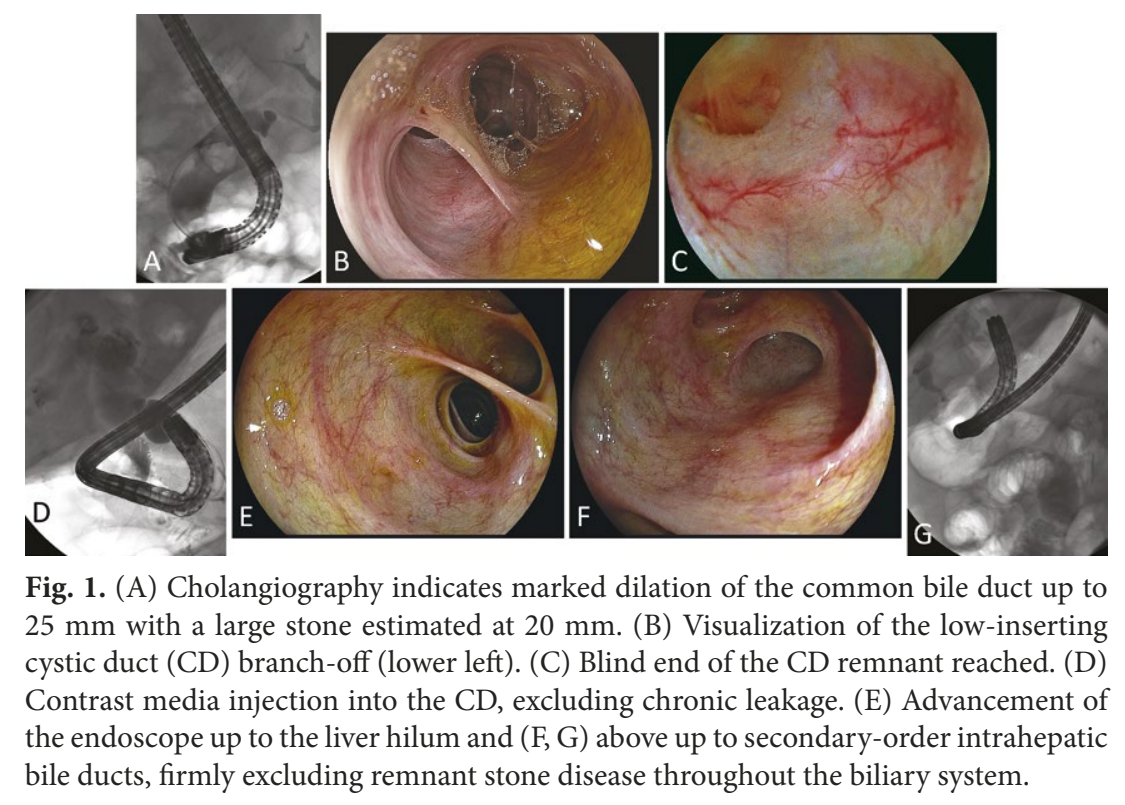


Procedural completeness of DC has been attributed to either advancement up to a predefined target region, e.g. indeterminate bile duct stricture, and/or up to the liver hilum [3]. Albeit persistent stone disease is not uncommon in largebore CD remnants, full direct ductoscopy of the (residual) CD and/or up to the spiral valves of Heister commonly precluding endoscopic passage might be discussed as a sine qua non for defining completion of full DC $[4,5]$.

\section{Vincent Zimmer}

Department of Medicine, Marienhausklinik St. Josef Kohlhof, Neunkirchen; Department of Medicine II, Saarland University Medical Center, Saarland University, Homburg, Germany

Correspondence: Vincent Zimmer, vincent.zimmer@gmx.de

Conflicts of interest: None.

DOI: $10.15403 /$ jgld-3714

\section{REFERENCES}

1. Baillie J. Biliary stenting in the management of large or multiple common bile duct stones. Gastrointest Endosc 2011;73:644. doi:10.1016/j. gie.2010.06.038

2. Zimmer V, Lammert F. Direct cholangioscopic visualization of pure „stento-mechanical“ lithotripsy. Dig Liver Dis 2020 Sep 29 doi:10.1016/j.dld.2020.09.016

3. ASGE Technology Committee, Komanduri S, Thosani N, et al. Cholangiopancreatoscopy. Gastrointest Endosc 2016;84:209-221. doi:10.1016/j.gie.2016.03.013

4. Zimmer V. Ultraslim cystic remnant ductoscopy for stone clearance under direct endoscopic vision. Gastrointest Endosc 2020;92:1268-1269. e1. doi:10.1016/j.gie.2020.06.018

5. Zimmer V. Spiral valves of Heister as visualized by direct cystic ductoscopy. Gastrointest Endosc 2018;87:1587-1589. doi:10.1016/j. gie.2017.11.020

\section{Impact of solid necrotic content on the outcome of endoscopic ultrasound guided transmural drainage of walled off pancreatic necrosis}

\section{To the Editor,}

We read with great interest the study of Seicean et al. [1] regarding the impact of solid necrotic content on the outcome of endoscopic ultrasound (EUS) guided transmural drainage (ETD) of walled off necrosis (WON). They reported that solid necrotic content of WON was an important determinant for the number of direct endoscopic necrosectomy (DEN) sessions and thereby, for the resolution time for endoscopic drainage. WON with higher solid debris content (> 50\%) were associated with a higher number of DEN, required longer endoscopy time and incurred higher costs [1]. Pancreatic fluid collections (PFC's) following acute necrotizing pancreatitis (ANP) have varying morphology and thus differ in their size, extent, and proportion of solid necrotic content. ETD of WON ushered a new era of minimally invasive treatment of organized pancreatic necrosis. The critical issue in ETD is to provide a transmural tract of sufficient diameter that allows drainage of thick necrotic debris as well as the passage of endoscope into the necrotic cavity for a possible DEN. The advent of large diameter lumen apposing metal stent (LAMS) has revolutionized the ETD of PFC's as these stents are easier to deploy with high success rates and, if required, DEN can be easily performed through these large caliber stents without need of repeated stent exchanges [2].

We have also previously demonstrated that the proportion of solid necrotic material in WON, as measured by EUS, can predict the need of DEN and other aggressive endoscopic drainage interventions with patients having $>40 \%$ solid debris on EUS requiring either DEN or surgical necrosectomy [3]. Also, the timing of intervention after the onset of ANP can determine the need of aggressive endoscopic interventions such as DEN. In a recent study comparing early ETD (within 4 weeks of onset of ANP) with delayed ETD ( $\geq 4$ weeks of onset of ANP), we found that DEN was required more frequently in the early group (50\% vs $7.4 \%$; $<<0.001$ ) [4]. The results of this study suggested that the timing of drainage is critical in determining the solid content of the PFC's and hence the efficacy of ETD.

Seicean et al. [1] performed DEN in $84.7 \%$ patients with WON. DEN can effectively remove solid necrotic debris and can hasten clinical success. However, DEN is associated with an increased risk of serious adverse events with high morbidity and mortality along with being labor intensive with higher costs [2]. Previous studies demonstrated that the majority of patients with WON probably do not need DEN and various alternative techniques such as nasocystic tube placement, irrigation by hydrogen peroxide and creating multiple transluminal gateways for drainage can improve the outcomes of ETD without resorting to DEN. Therefore, a "step-up" endoscopic approach has been advocated with DEN being reserved for patients not responding to aggressive drainage and irrigation [5].

The main conclusion inferred from this study as well as our previous studies is that the need for DEN in patients with WON depends upon the proportion of the solid necrotic debris content. The threshold of the solid necrotic content that predicts the need for DEN is variable across studies because of the varying use of step-up technique endoscopic drainage and lack of objectivity in accurately quantifying the solid necrotic content. Thus, there is a need to develop a uniform objective method of determining the exact solid necrotic content in WON and newer imaging modalities like 3-D EUS may help in better quantifying the solid necrotic content.

\section{Nikhil Bush ${ }^{1}$, Surinder Singh Rana ${ }^{1}$, Rajesh Gupta ${ }^{2}$ \\ 1) Department of Gastroenterology, Postgraduate Institute of Medical Education and Research (PGIMER), Chandigarh; 2) Department of Surgical Gastroenterology, Postgraduate Institute of Medical Education and Research (PGIMER), Chandigarh, India}

Correspondence: Prof. Surinder S. Rana, drsurinderrana@yahooco.in

Conflicts of interest: None.

DOI: $10.15403 /$ jgld-3777 


\section{REFERENCES}

1. Seicean A, Pojoga C, Moștean O, et al. What is the impact of the proportion of solid necrotic content on the number of necrosectomies during eus-guided drainage using lumen-apposing metallic stents of pancreatic walled-off necrosis? J Gastrointestin Liver Dis 2020;29:623628. doi:10.15403/jgld-3128

2. Rana SS. An overview of walled-off pancreatic necrosis for clinicians. Expert Rev Gastroenterol Hepatol 2019;13:331-343. doi:10.1080/174 74124.2019.1574568

3. Rana SS, Bhasin DK, Sharma RK, Kathiresan J, Gupta R. Do the morphological features of walled off pancreatic necrosis on endoscopic ultrasound determine the outcome of endoscopic transmural drainage? Endosc Ultrasound 2014;3:118-122. doi:10.4103/2303-9027.131039

4. Rana SS, Sharma R, Kishore K, Dhalaria L, Gupta R. Safety and Efficacy of Early (<4 Weeks of Illness) Endoscopic Transmural Drainage of Post-acute Pancreatic Necrosis Predominantly Located in the Body of the Pancreas. J Gastrointest Surg 2021 Feb 5. doi:10.1007/s11605-02104945-y

5. Rana SS, Sharma V, Sharma R, Gupta R, Bhasin DK. Endoscopic ultrasound guided transmural drainage of walled off pancreatic necrosis using a „step - up“ approach: A single centre experience. Pancreatology 2017;17:203-208. doi:10.1016/j.pan.2017.02.007

\section{Durable response with lenvatinib and pembrolizumab combination therapy in a patient with pre-treated metastatic cholangiocarcinoma}

\section{To the Editor,}

We read with interest the article of Cosma et al. [1] who described the efficacy of lenvatinib for patients with hepatocellular carcinoma and impaired liver function. Whether lenvatinib is effective for other hepatobiliary cancers is yet unclear. Here, we present the case of a patient with unresectable intrahepatic cholangiocarcinoma (iCCA) who achieved substantial und durable response from combination therapy with lenvatinib and pembrolizumab despite intensive pre-treatment.

A 59-year-old male patient was referred to our department for workup of a liver tumor. Diagnosis of alcoholic liver cirrhosis (Child-Pugh score A, MELD score 9) was made based on the patient's history and diagnostic findings. An abdominal magnetic resonance imaging (MRI) indicated a hypervascular lesion of $4.3 \times 3.9 \mathrm{~cm}$ diameter in liver segment VIII and multiple smaller lesions in segments VII and VIII (Fig. 1A). Furthermore, a peripancreatic lymphadenopathy was detected (Fig. 1B). Histopathological examination of the lesion was consistent with an adenocarcinoma of pancreaticobiliary origin (Fig. 1C). Combining medical imaging and histopathology, diagnosis of a metastatic iCCA was made. The TNM classification was cT2, cN1, cM1.

Due to the multifocality of the tumour and lack of surgical therapeutic options, palliative chemotherapy with gemcitabine and cisplatin was initiated and continued for 10 months until progressive mediastinal lymphadenopathy occurred. The patient was subsequently enrolled in the open label, phase II NALIRICC trial (NCT03043547), and received irinotecan liposome, 5-fluorouracil and leucovorin. As progressive lymphogenic and hepatic disease was detected 6 months later, panel sequencing of the tumour genome was performed, revealing only a TP53 p.Y236N mutation. Hence, a combination of lenvatinib and pembrolizumab was initiated as an off-label treatment (lenvatinib at $10 \mathrm{mg}$ p.o. daily and pembrolizumab at $200 \mathrm{mg}$ i.v. every 3 weeks).

A CT scan in 5 months later revealed partial response with reduction of lymphogenic and hepatic tumor manifestations,
A

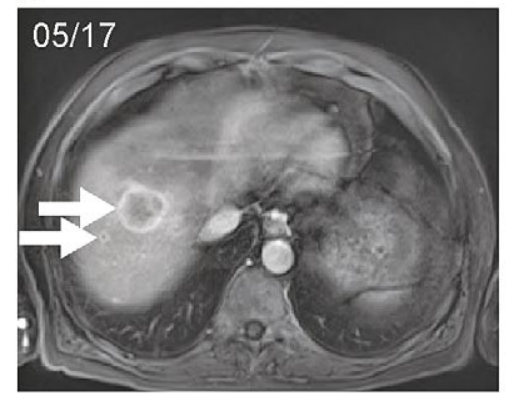

B

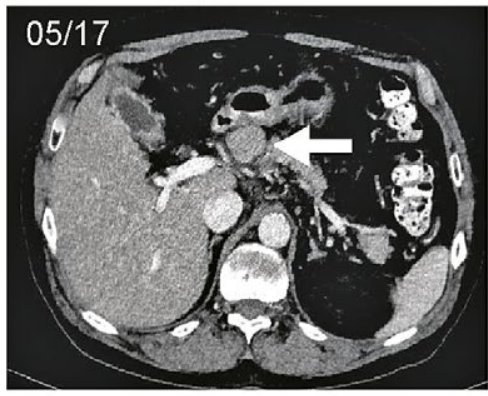

C

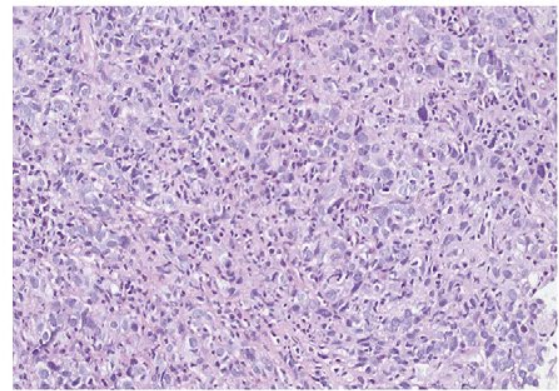

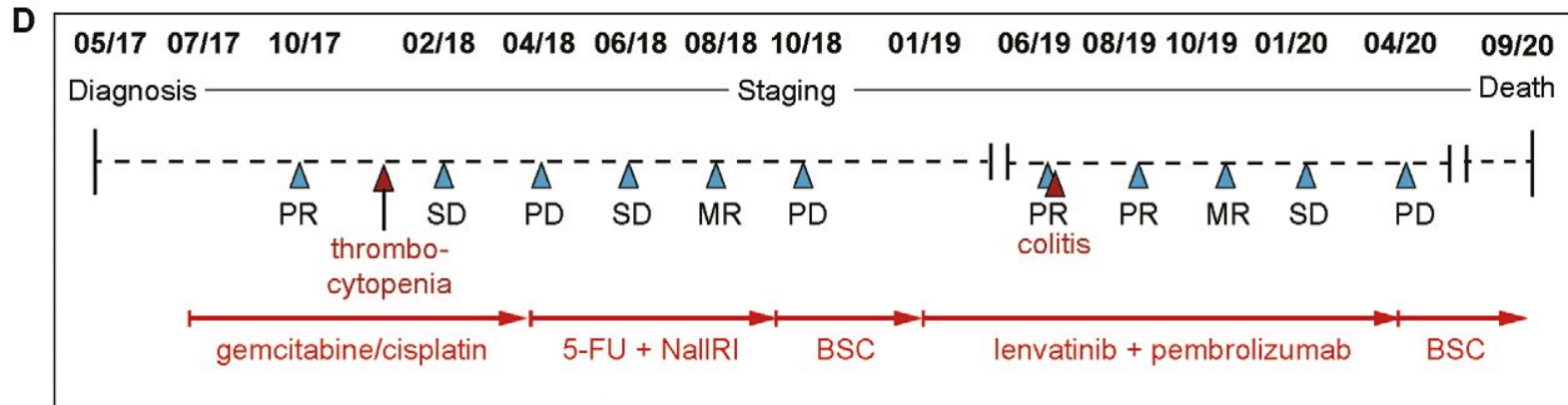

Fig. 1. A: Abdominal magnetic resonance imaging: hepatic lesions at time of diagnosis. B: Computer tomography scan showing peripancreatic lymphadenopathy. C: Histopathological examination: adenocarcinoma of pancreaticobiliary origin. D: Time line of treatment course. PR: partial response; SD: stable disease; PD: progressive disease; MR: mixed response; BSC: best supportive care. 
mixed response two months later and consecutive stable disease. Adverse effects experienced with lenvatinib/ pembrolizumab were pruritus that resolved after a few weeks and occasional hypertension. Five months after treatment initiation the patient developed an immune-checkpoint inhibitor induced colitis and gastritis. Treatment with oral prednisolone and pantoprazole was installed, and therapy with lenvatinib/pembrolizumab was continued. Symptoms resolved within two weeks and prednisolone was tapered. Fifteen months after treatment initiation, progression of lymphogenic and hepatic lesions and new adrenal manifestations occurred and therapy with lenvatinib/pembrolizumab was discontinued. The patient opted for best supportive care and deceased subsequently. Overall survival after diagnosis was 40 months and 21 months after initiation of lenvatinib/pembrolizumab treatment (Fig. 1D).

In CCA, evidence regarding efficacy of lenvatinib is limited. Ueno et al. [2] reported antitumor activity of lenvatinib as a monotherapy in a single-arm, open-label phase II trial comprising 26 patients with unresectable CCA and prior failure of response to chemotherapy. Objective response rate (ORR) in this study was $11.5 \%$, with partial response in three patients and stable disease achieved in nine patients [2]. There is increasing evidence that the addition of immune checkpoint inhibitors (ICI) to multikinase inhibitors such as lenvatinib can enhance antitumor efficacy [3, 4]. In the ongoing phase II LEAP-005 trial, the combination of lenvatinib/pembrolizumab is assessed in a multicohort design, and 31 patients with CCA have been enrolled (NCT03797326). The ORR for CCA was $10 \%$ and disease control rate was (DCR) 68\% [5]. Recently, Lin et al. [6] reported the use of lenvatinib/pembrolizumab in a single-arm study with 32 patients with CCA as nonfirst-line treatment. In this study, 16 patients with iCCA, 10 with extrahepatic CCA and 6 with gall bladder cancer were included. The median progression-free survival was 4.9 months with an ORR of $25 \%$, and DCR of $78.1 \%$. These results indicate a promising antitumor activity of lenvatinib/ pembrolizumab in CCA, but results from larger randomised, controlled trials are lacking.

In conclusion, combination therapy with lenvatinib/ pembrolizumab can be an effective treatment option in patients with CCA even with extensive pre-treatment. Results from ongoing clinical trials with this drug combination will allow a more comprehensive assessment of its clinical efficiency and safety profile for patients with CCA. Finally, predictive biomarkers are required to identify patients who benefit from treatment with lenvatinib/pembrolizumab.

Lena Dreikhausen ${ }^{1}$, Alexander Kusnik ${ }^{1,2}$, Nadine Schulte ${ }^{1}$, Maximilian Eckardt ${ }^{1}$, Andreas Teufel ${ }^{1,2}$, Timo Gaiser ${ }^{3}$, Matthias Ebert $^{1,2}$, Tianzuo Zhan ${ }^{1}$

1) Department of Medicine II, University Medical Center Mannheim, Medical Faculty Mannheim, Heidelberg University, Mannheim;

2) Preventive Medicine Baden-Württemberg (CPMBW), Medical Faculty Mannheim, Heidelberg University; 3) Institute of Pathology, University Medical Center Mannheim, Medical Faculty Mannheim, Heidelberg University, Mannheim, Germany

Correspondence: Tianzuo Zhan, MD, tianzuo.zhan@umm.de
Conflicts of interest: None.

Acknowledgements: L.D. and T.Z. were supported by the Clinician Scientist program "Interfaces and Interventions in Chronic Complex Conditions" funded by the DFG (EB 187/8-1).

DOI: $10.15403 /$ jgld-3730

\section{REFERENCES}

1. Cosma LS, Weigand K, Müller-Schilling M, Kandulski A. Lenvatinib as First-line Treatment of Hepatocellular Carcinoma in Patients with Impaired Liver Function in Advanced Liver Cirrhosis: Real World Data and Experience of a Tertiary Hepatobiliary Center. J Gastrointestin Liver Dis 2021;30:247-253. doi:10.15403/jgld-3345

2. Ueno M, Ikeda M, Sasaki T, et al. Phase 2 study of lenvatinib monotherapy as second-line treatment in unresectable biliary tract cancer: primary analysis results. BMC Cancer 2020;20:1105 doi:10.1186/s12885-020-07365-4

3. Galon J, Bruni D. Approaches to treat immune hot, altered and cold tumours with combination immunotherapies. Nat Rev Drug Discov 2019;18:197-218. doi:10.1038/s41573-018-0007-y

4. Shigeta K, Datta M, Hato T, et al. Dual Programmed Death Receptor-1 and Vascular Endothelial Growth Factor Receptor-2 Blockade Promotes Vascular Normalization and Enhances Antitumor Immune Responses in Hepatocellular Carcinoma. Hepatology 2020;71:1247-1261. doi:10.1002/hep.30889

5. Taylor MH, Schmidt EV, Dutcus C, et al. The LEAP program: lenvatinib plus pembrolizumab for the treatment of advanced solid tumors. Future Oncol 2021;17:637-648. doi:10.2217/fon-2020-0937

6. Lin J, Yang X, Long J, et al. Pembrolizumab combined with lenvatinib as non-first-line therapy in patients with refractory biliary tract carcinoma. Hepatobiliary Surg Nutr 2020;9:414-424. doi:10.21037/hbsn-20-338

\section{Use of a novel viscous lifting solution $\left(\mathrm{Lift} \mathrm{Up}^{\circledR}\right)$ to enable safer and larger en bloc endoscopic mucosal resection in deep enteroscopy}

\section{To the Editor}

We present a case of a 29 year-old female patient with Peutz Jeghers syndrome (PJS) referred to our unit for endoscopic mucosal resection (EMR) of a large small bowel polyp. The diagnosis of PJS was recently established by the presence of hyperpigmented macules on the lips and oral mucosa and the resection of a hamartomatous polyp on a previous colonoscopy. A prior capsule endoscopy study revealed a large polyp in the jejunum. A previous deep enteroscopy was performed at another institution, where a $60 \mathrm{~mm}$ sessile polyp in the proximal jejunum was confirmed and an endoloop was placed around a portion of the polyp to decrease the polyp size.

At our unit, anterograde single-balloon enteroscopy was repeated to reevaluate and resect the larger polyp, which at the time measured around $40 \mathrm{~mm}$. Submucosal fibrosis resulting from the previous endoloop was identified during initial submucosal lifting with saline plus methylene blue, therefore the lifting solution was switched to Liftup ${ }^{\oplus}$ (Ovesco, Tübingen Germany), a viscous solution, which enabled proper 


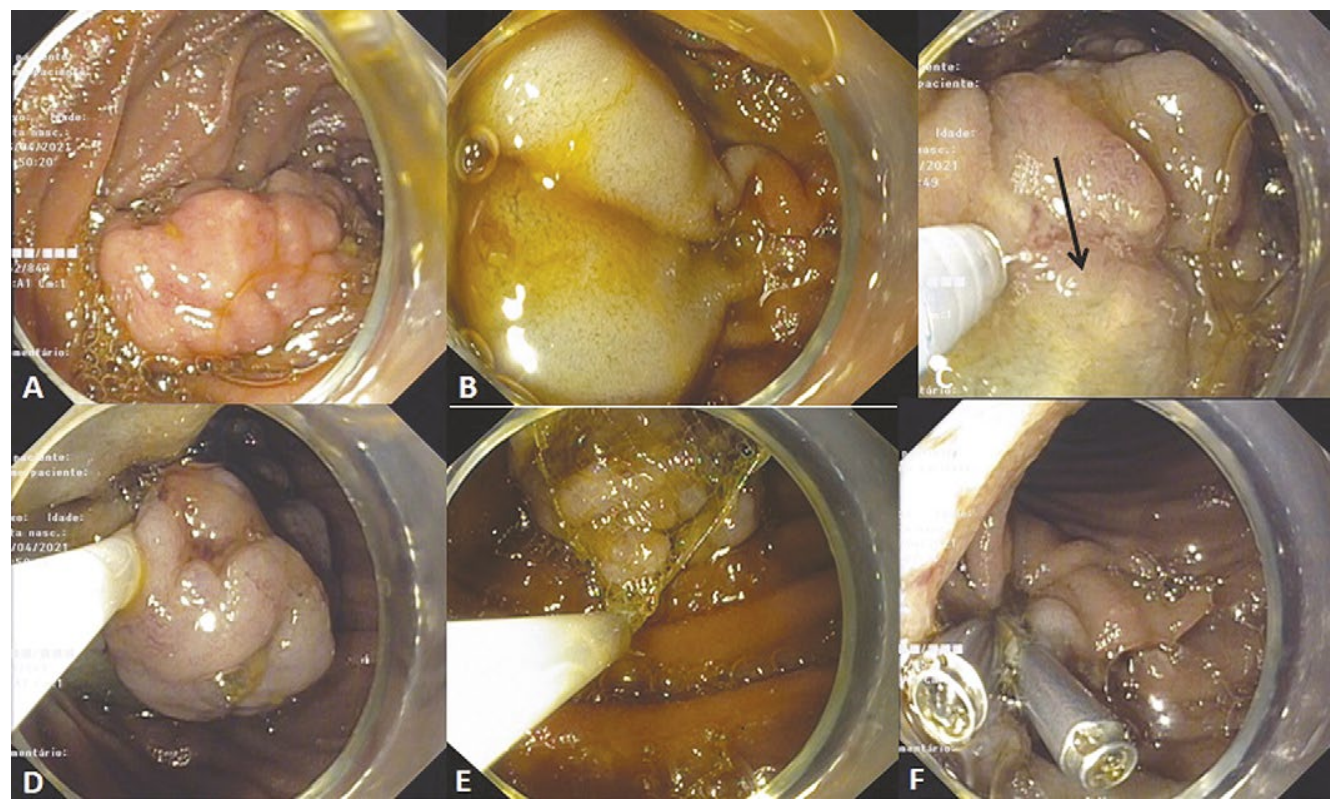

Fig. 1. Endoscopic mucosal resection of a large small-bowel polyp: A) Small-bowel polyp; B) Submucosal elevation after Liftup injection; C) Submucosal fibrosis resulting from the previous endoloop was identified during initial submucosal lifting; D) Polyp snaring; E) Polyp retrieval with a Roth Net; F) Closure of the scar with clips.

lifting. En bloc EMR was then safely completed and the scar prophylactically closed with 4 clips. Histology confirmed a hamartomatous polyp without dysplasia. No immediate or late complications occurred and the patient was discharged after 48 hours of inpatient monitoring.

Patients with PJS are often diagnosed with small bowel polyps, some of them large, most of them being successfully managed with deep enteroscopy [1-3]. LiftUp ${ }^{2}$ serves as a viscous injection solution for endoscopic resection techniques. It is a thermoreversible agent at body temperature, containing ethylenoxide and propylenoxid, and forms a stable and long-lasting cushion in the submucosa, which has been associated with almost no procedure related perforations $[4,5]$. Wedi et al. [4], conducted a prospective randomized, ex vivo study which compared the use of three different solutions, Lifup ${ }^{\circ}$, hydroxyethyl starch (HAES 6\%) and normal saline solution $(\mathrm{NaCl} 0.9 \%)$ before endoscopic mucosal dissection (ESD). The authors found higher adequate lifting rates with $\mathrm{LiftUp}^{\circ}$ (80\% vs. $30 \%$ and $30 \%$, $\mathrm{p}<0.002)$ and significantly less injected volume of this agent ( $33.5 \mathrm{~mL}$ vs. $231 \mathrm{~mL}$ and $299 \mathrm{~mL}, \mathrm{p}<0.001$ ) [4]. The aim of this case-report is to emphasize the use of LiftUp solution to lift a fibrotic small bowel lesion, enabling a safe en bloc EMR of a large small bowel polyp.

Catarina Gomes ${ }^{1}$, Rolando Pinho ${ }^{1}$, Rita Costa ${ }^{2}$, Pedro Antunes ${ }^{2}$, Ana Ponte ${ }^{1}$, Adélia Rodrigues ${ }^{1}$

1) Gastroenterology department, Centro Hospitalar Vila Nova de Gaia/ Espinho; 2) Gastroenterology department, Hospital de Braga, Portugal

Correspondence: Ana Catarina Ribeiro Gomes, catarina.rib.gomes@gmail.com

Conflicts of interest: None.

DOI: $10.15403 /$ jgld-3822

\section{REFERENCES}

1. Serrano M, Mão-de-Ferro S, Pinho R, et al. Double-balloon enteroscopy in the management of patients with Peutz-Jeghers syndrome: a retrospective cohort multicenter study. Rev Esp Enferm Dig 2013;105:594-599. doi:10.4321/s1130-01082013001000004

2. Pinho R, Mascarenhas-Saraiva M, Mão-de-Ferro S, et al. Multicenter survey on the use of device-assisted enteroscopy in Portugal. United European Gastroenterol J 2016;4:264-274. doi:10.1177/2050640615604775

3. Pérez-Cuadrado-Robles E, Pinho R, Gonzalez B, et al. Small Bowel Enteroscopy - A Joint Clinical Guideline from the Spanish and Portuguese Small Bowel Study Groups. GE Port J Gastroenterol 2020;27:324-335. doi:10.1159/000507375

4. Wedi E, Koehler P, Hochberger J, et al. Endoscopic submucosal dissection with a novel high viscosity injection solution (LiftUp) in an ex vivo model: a prospective randomized study. Endosc Int Open 2019;7:E641-E646. doi:10.1055/a-0874-1844

5. Wedi E, Ho CN, Conrad G, et al. Preclinical evaluation of a novel thermally sensitive co-polymer (LiftUp) for endoscopic resection. Minim Invasive Ther Allied Technol 2019;28:277-284. doi:10.1080/13645706.2018.1535440

\section{Pembrolizumab induced steroid resistant immune mediated colitis and concurrent Clostridioides difficile infection}

\section{To the Editor,}

Pembrolizumab, a monoclonal antibody might induce gastrointestinal side effects such as diarrhea and immune mediated colitis with intestinal perforation and important dehydration [1].

We herein present a case of pembrolizumab induced colitis and concurrent Clostridioides difficile infection in a 
patient with non-small-cell lung cancer. A 53-year-old male, presented to emergency department for severe diarrhea (about 10 stools per day with blood) and altered status. In April 2020 he was diagnosed with a left central hilar pulmonary tumor and mediastinal lymphadenopathy. Immunohistochemistry showed the presence of adenocarcinoma epidermal growth factor receptor (EGFR) negative, anaplastic lymphoma kinase (ALK) negative and PD-L1 80\%. He had 11 infusions with Pembrolizumab monotherapy, with significant regression of pulmonary nodules on a computed tomography exam. Before the last dose of Pembrolizumab the patient presented blood in stool and liquid bowel movements; a sigmoidoscopy was performed that revealed circumferential edema, friability, spontaneous bleeding, punctiform ulcerations along the first $40 \mathrm{~cm}$ from the AO. There were performed multiple biopsies and an immune mediated colitis induced by Pembrolizumab treatment was suspected. The patient was tested negative for Clostridioides difficile toxins, and a stool culture was negative; therefore, he began steroid-therapy with Prednisolone 32 mg/day (February 2021) without any improvement. After 2 weeks the patient was admitted to our department for severe diarrhea (about 10 stools per day with blood). This time, the Clostridioides difficile polymerase chain reaction (PCR) test for toxin A/B were positive. Vancomycin $250 \mathrm{mg}$ every 6 hours and Metronidazole $500 \mathrm{mg}$ every 8 hours was initiated, and Prednisolone $32 \mathrm{mg} /$ day was maintained. As partial response was achieved, Tigecycline $50 \mathrm{mg}$ every 12 hours intra-venously was initiated, without clinical response. In the $7^{\text {th }}$ day from the admission, he had a colonoscopy, that revealed features of severe inflammation with marked erythema, friability, spontaneous bleeding and ulcerations from ascending colon to rectum; ileocecal valve mucosa with normal appearance (Fig. 1A) The histopathological examination showed diffuse mucosal inflammation, architectural distortion with ramification and shortening of the crypts, basal plasmacytosis (arrow) and cubic, regenerative surface epithelium confirming the diagnosis of immune mediated colitis (Fig. 1B). Tumor necrosis factor (TNF) alpha antagonist treatment (Infliximab $5 \mathrm{mg} / \mathrm{kg}$ ) and mesalazine therapy $1000 \mathrm{mg}$ three times per day was added to prednisolone (total duration of prednisolone therapy 6 weeks). After the first two doses of Infliximab the patient presented an improvement of clinical manifestations with 4-5 stools/day, without blood or

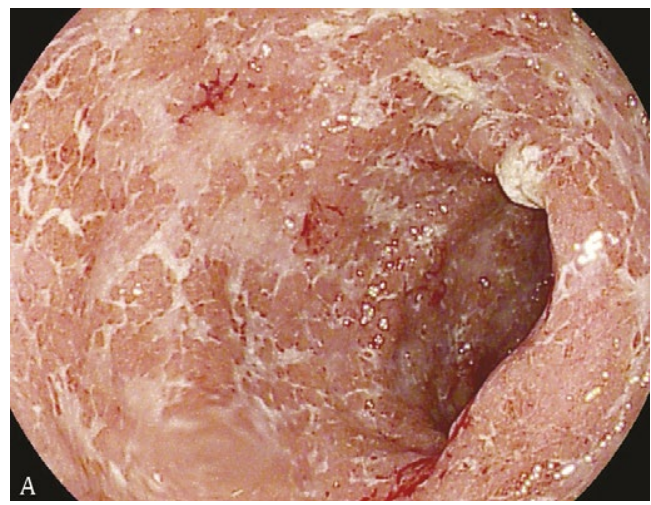

Fig. 1A. Diagnostic colonoscopy revealing severe inflamation with marked erythema, friability, spontaneous bleeding, ulcerations of sigmoid and rectum.

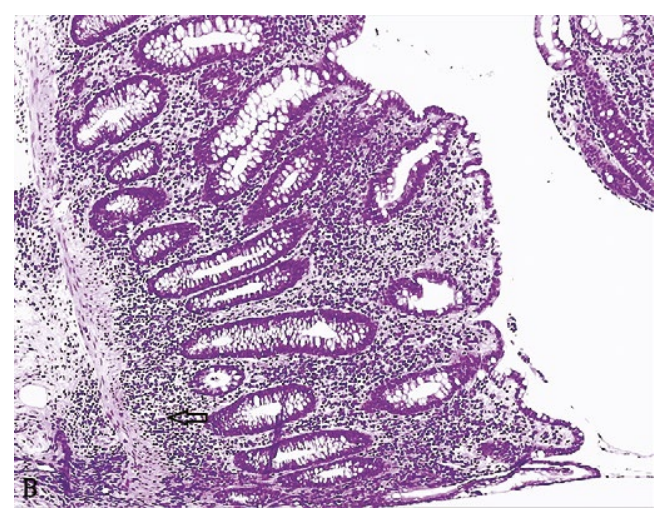

Fig. 1B. Diffuse mucosal inflammation, architectural distortion with ramification and shortening of the crypts, basal plasmacytosis (arrow) and cubic, regenerative surface epithelium.

mucus. For Clostridioides difficile superinfection resistant to Vancomycin, Metronidazole and Tigecycline, patient received fecal microbiota transplantation, through a naso-gastric tube. This FMT led to the complete resolution of the symptoms. We continued Infliximab $5 \mathrm{mg} / \mathrm{kg}$ every 2 weeks (three more infusions), until the fecal calprotectin was normal.

This case is particular because of concomitant immune mediated colitis (steroid-refractory) with Clostridioides difficile superinfection (resistant to Vancomycin, Metronidazole and Tigecycline) successfully treated with Infliximab $5 \mathrm{mg} / \mathrm{kg}$ every 2 weeks and fecal microbiota transplantation. Cases of steroid-refractory immune-mediated colitis successfully treated either with one dose of Infliximab $(5 \mathrm{mg} / \mathrm{kg}$ ) either with two or more doses $(5-10 \mathrm{mg} / \mathrm{kg}$ ) were previously reported; there were reported cases with resistance to Infliximab [2, 3]. Two cases described the association between immune mediated colitis and Clostridioides difficile infection, but both of them responded to corticosteroids and antibiotics $[4,5]$.

Colonoscopy and multiple colonic biopsies are necessary for the diagnosis of immune-induced colitis; the endoscopic and histological aspects are mimicking inflammatory bowel diseases but lacks chronic architectural changes of the crypts $[4,5]$.

Carmen Monica Preda ${ }^{1}$, Raluca Ioana Marin ${ }^{2}$, Gabriel Becheanu ${ }^{3}$, Florina Neacsu ${ }^{3}$, Teodora Manuc ${ }^{1}$, Mircea Manuc ${ }^{1}$

1) Carol Davila University of Medicine and Pharmacy, Gastroenterology \& Hepatology Department, Fundeni Clinic Institute, Bucharest; 2) Gastroenterology \& Hepatology Department, Fundeni Clinic Institute, Bucharest; 3) Carol Davila University of Medicine and Pharmacy, Morphopathology Department, Fundeni Clinic Institute, Bucharest, Romania

Correspondence: Carmen Monica Preda, carmenmonica.preda@gmail.com

Conflicts of interest: None.

DOI: $10.15403 /$ jgld-3923

\section{REFERENCES}

1. Bellaguarda E, Hanauer S. Checkpoint Inhibitor-Induced Colitis. Am J Gastroenterol 2020;115:202-210. doi:10.14309/ajg.0000000000000497 
2. Nahar KJ, Rawson RV, Ahmed T, et al. Clinicopathological characteristics and management of colitis with antiPD1 immunotherapy alone or in combination with ipilimumab. J Immunother Cancer 2020;8:e001488. doi:10.1136/jitc-2020-001488

3. Paparoupa M, Stupperich S, Goerg-Reifenberg L, Wittig A, Schuppert F. Successful treatment of an immune-mediated colitis induced by checkpoint inhibitor therapy in a patient with advanced melanoma Case Rep Gastroenterol 2020;14:554-560. doi:10.1159/000511252
4. Zhou C, Klionsky Y, Treasure ME, Bruno DS. PembrolizumabInduced Immune-Mediated Colitis in a Patient with Concurrent Clostridium Difficile Infection. Case Rep Oncol 2019;12:164-170. doi:10.1159/000497155

5. Gupta A, Khanna S. Ipilimumab-associated colitis or refractory Clostridium difficile infection? BMJ Case Rep 2015;2015:bcr2015211160. doi:10.1136/bcr-2015-211160 